\title{
Atropine-Induced Ventricular Asystole During a Dobutamine Stress Test
}

\author{
Kevin G. Buda ${ }^{\text {a, c }}$, Joseph D. Steffens ${ }^{\mathrm{a}}$, Abdulrahman Gamamª, Shrikar Rajagopal ${ }^{\mathrm{a}}$, \\ Fredrick Ogugua $^{\mathrm{a}}$, Jorge L. Reyes ${ }^{\mathrm{a}}$, Rehan Karim ${ }^{\mathrm{b}}$, Charles A. Herzog ${ }^{\mathrm{b}}$
}

\begin{abstract}
Dobutamine stress echocardiography is a safe diagnostic test with low incidence of serious complications. Atropine has been shown to reduce test duration without increasing the rate of complications. We present a case of a 52-year-old man with end stage renal disease who experienced syncope due to ventricular asystole after atropine administration during a dobutamine stress test. The underlying pathophysiology is discussed.
\end{abstract}

Keywords: Atropine; Asystole; Dobutamine stress echocardiography; Transthoracic echocardiography

\section{Introduction}

Dobutamine stress echocardiography (DSE) is frequently used to diagnose coronary artery disease, evaluate valvular heart disease, and risk-stratify patients prior to non-cardiac surgery. It is a safe procedure with serious adverse events occurring in less than $1 \%$ of cases $[1,2]$. Atropine is used during DSE to reduce test duration without increasing the rates of complications [3]. Ventricular asystole is a rare complication, occurring in $<0.01 \%$ of cases $[4,5]$. We present a case of transient third-degree heart block following atropine administration during DSE resulting in ventricular asystole. To the best of our knowledge, this is the first report of atropine-induced ventricular asystole during a dobutamine stress test.

\section{Case Report}

A 52-year-old man with end stage renal disease (ESRD), dia-

Manuscript submitted April 5, 2020, accepted April 13, 2020

a Department of Internal Medicine, Hennepin Healthcare, Minneapolis, MN, USA

${ }^{b}$ Cardiology Division, Department of Internal Medicine, Hennepin Healthcare, University of Minnesota, Minneapolis, MN, USA

${ }^{\mathrm{c} C o r r e s p o n d i n g ~ A u t h o r: ~ K e v i n ~ G . ~ B u d a, ~ D e p a r t m e n t ~ o f ~ I n t e r n a l ~ M e d i c i n e, ~}$ Hennepin County Medical Center, 701 Park Avenue, G5-125, Minneapolis, MN 55401, USA. Email: kevin.buda@hcmed.org

doi: https://doi.org/10.14740/jmc3465 betes mellitus, peripheral vascular disease, and essential hypertension on carvedilol presented to the echocardiography lab for cardiovascular risk stratification for renal transplant evaluation. At rest, echocardiography showed an ejection fraction of $65 \%$ and no wall motion abnormalities. The initial electrocardiogram (ECG) showed no evidence of conduction system abnormalities (Fig. 1a). Twenty seconds after administration of the second $0.2 \mathrm{mg}$ dose of atropine, the PR interval prolonged and the patient developed a complete heart block with a 15-s episode of ventricular asystole due to persistent non-conduction of $\mathrm{P}$ waves without ventricular escape (Fig. 2a). The atrial rate did not increase during this episode. The patient lost consciousness for approximately $10 \mathrm{~s}$ and denied any symptoms on awakening. During stress, echocardiography showed a wall motion abnormality of the distal septum and anterior apex (present on stress echocardiography 2 years prior), and the ECG did not show ischemic changes. Echocardiography immediately after the syncopal episode showed a decrease in ejection fraction from $62 \%$ to $45 \%$ that resolved after $75 \mathrm{~min}$.

The patient was admitted and $2 \mathrm{~h}$ after the initial episode of syncope, telemetry captured another 13.5-s period of ventricular asystole with persistent non-conduction of $\mathrm{P}$ waves without ventricular escape or a compensatory increase in atrial rate (Fig. 2b). The patient did not lose consciousness and endorsed only lightheadedness. Repeat ECG showed first-degree atrioventricular (AV) block (Fig. 1b). The patient's carvedilol was held given the unknown etiology of the heart block. Electrolytes were within normal limits. Urgent coronary angiography did not show any obstructive coronary disease. An electrophysiology study was performed to assess $\mathrm{AV}$ nodal conduction in response to atropine administration. There was no evidence of infra-His conduction block with incremental atrial pacing, nor was there any HV interval change with multiple successive doses of atropine for a total of $1 \mathrm{mg}$.

The patient had no further episodes of heart block during his admission. He resumed his home medications and was discharged with the presumed diagnosis of atropine-induced bradycardia and heart block with an ambulatory cardiac monitor that showed sinus rhythm with a PR interval of 218 $\mathrm{ms}$. There were two short runs of supraventricular tachycardia (SVT), the longest lasting for 13 beats, and one episode of high-grade AV block resulting in a 3.1-s pause with three consecutive non-conducted $\mathrm{P}$ waves. Due to the asymptomatic nature of this pause, no pacemaker was placed. 


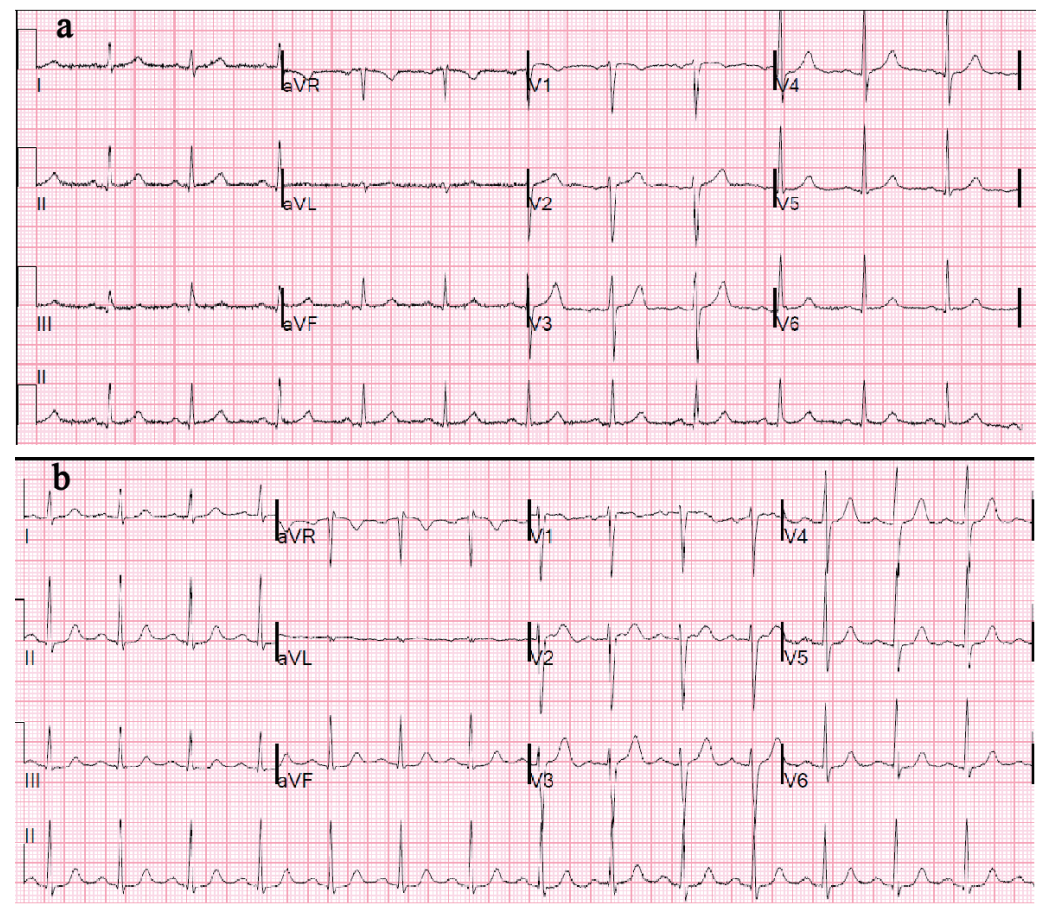

Figure 1. (a) Baseline ECG with NSR, LVH, and LAA, $P R=0.19 \mathrm{~s}$. (b) ECG after second episode of third-degree AV block (PR $=0.22 \mathrm{~s}$ ), which demonstrates new first-degree AVB. ECG: electrocardiogram; AVB: atrioventricular block; LVH: left ventricular hypertrophy; NSR: normal sinus rhythm.

\section{Discussion}

Ventricular asystole is a rare complication that can occur during DSE with an incidence of $0.002 \%$ [1]. Case reports have shown both dobutamine and atropine-mediated complete heart block [6]. In this case, the absence of chest pain, lack of ST changes on ECG, lack of occlusion on coronary angiography, and normal electrophysiology study suggest a non-ischemic and non-structural etiology of the patient's ventricular asystole. Pharmacokinetics also definitively ruled out dobutamine as the etiology for the patient's ventricular asystole, as ventricular asystole occurred outside its half-life ( $2 \mathrm{~min}$ ) but within the half-life of atropine $(3 \mathrm{~h})$. Therefore, the mechanism of third-degree AV block in this patient was most likely related to atropine-mediated hypervagotonia, suggested by the close temporal association between this rhythm and atropine administration, the second episode occurring within the half-life of atropine but not dobutamine, and the lack of a compensatory increase in SA nodal rate during ventricular asystole, consistent with hypervagotonia.

There are several mechanisms through which atropine can lead to hypervagotonia. The Bezold-Jarisch reflex occurs when increased myocardial contraction stimulates myocardial mechanoreceptors, resulting enhanced parasympathetic activity [1]. The resulting vagal storm may contribute to transient complete block in patients with preexisting conduction system disease or reduced cardiac output. Similarly, hypervagotonia can occur with low dose atropine administration as a reflex in response to significant peripheral vasodilation and hypotension that can occur [7], as well as due to direct central and sinoatrial (SA) nodal effects. Both low and high dose atropine administration have been shown to increase pacemaker muscarinic activity, thereby decreasing heart rate [8]. Low dose atropine may also disinhibit the vagus nerve by inhibiting muscarinic receptors in the central vagal nuclei, contributing to atropine's bradycardic effects [9], an effect which may have been potentiated by our patient's beta-blockade.

\section{Conclusions}

This case demonstrates transient complete AV block with no ventricular escape after administration of atropine during DSE in a patient with multiple comorbidities. Atropine can cause a vagally mediated reflex that can slow SA nodal conduction, potentiate $\mathrm{AV}$ nodal conduction, and rarely result in complete heart block. AV block and ventricular asystole are rare complications that should be recognized prior to DSE and atropine administration.

\section{Acknowledgments}

None to declare.

\section{Financial Disclosure}

None to declare. 

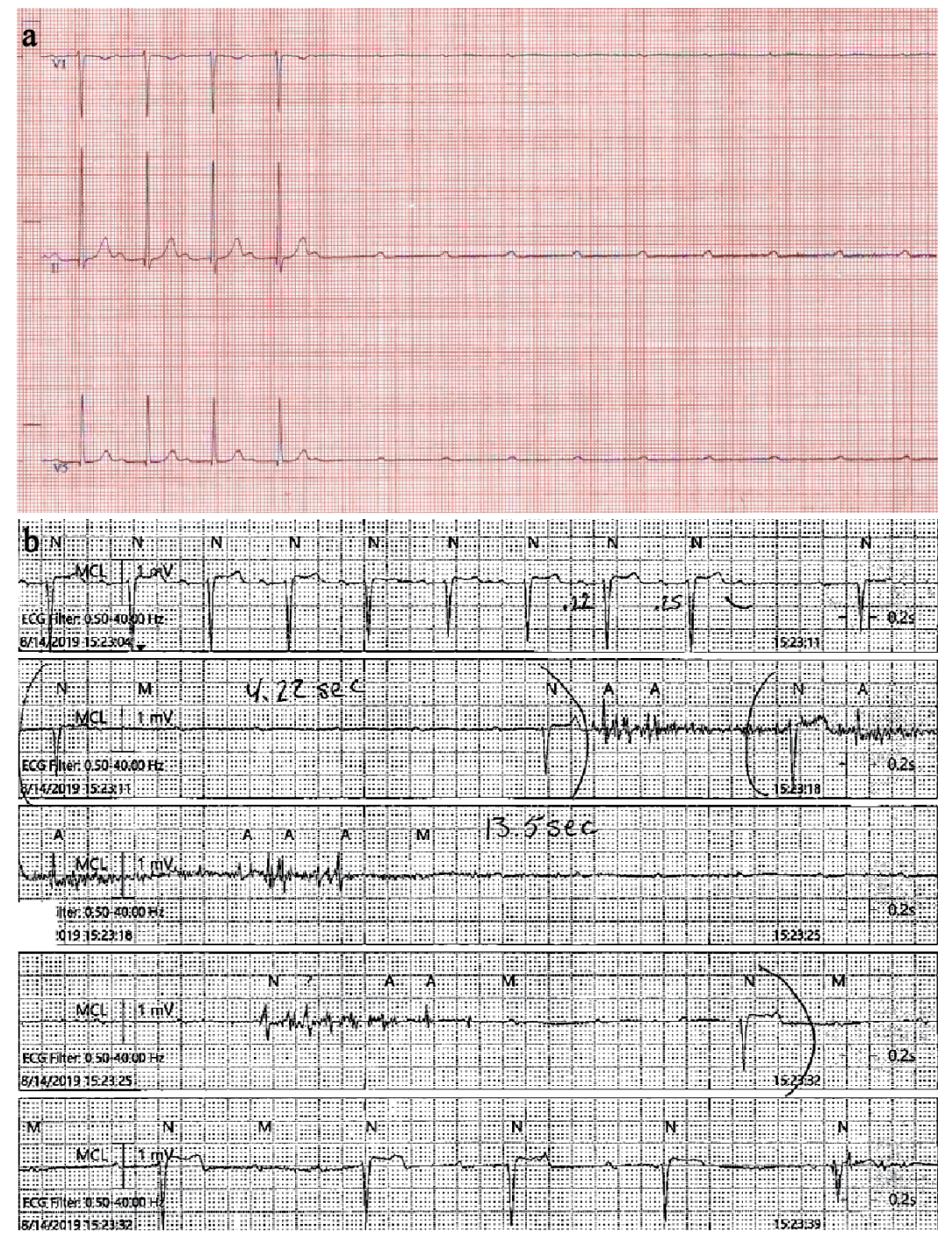

Figure 2. (a) ECG during DSE showing prolonging PR interval followed by complete heart block and ventricular asystole due to lack of escape rhythm. (b) Telemetry strip post-admission showing the same rhythm. ECG: electrocardiogram; DSE: dobutamine stress echocardiography.

\section{Conflict of Interest}

None to declare.

\section{Informed Consent}

Phone consent was obtained. The patient was agreeable to written consent, which was unable to be obtained at this time due to the COVID crisis.

\section{Author Contributions}

Kevin G. Buda: identification of case, data interpretation, literature review, drafting of article, critical revision of article, and approval of article; Joseph D. Steffens, Abdulrahman Gamam, Shrikar Rajagopal, Fredrick Ogugua, Jorge L. Reyes, Rehan Karim, and Charles A. Herzog: drafting of article, critical revision of article, and approval of article.

\section{Data Availability}

Any inquiries regarding supporting data availability of this study should be directed to the corresponding author.

\section{Abbreviations}

AV: atrioventricular; DSE: dobutamine stress echocardiography; ESRD: end stage renal disease; SVT: supraventricular tachycardia 


\section{References}

1. Geleijnse ML, Krenning BJ, Nemes A, van Dalen BM, Soliman OI, Ten Cate FJ, Schinkel AF, et al. Incidence, pathophysiology, and treatment of complications during dobutamine-atropine stress echocardiography. Circulation. 2010;121(15):1756-1767.

2. Mertes H, Sawada SG, Ryan T, Segar DS, Kovacs R, Foltz J, Feigenbaum H. Symptoms, adverse effects, and complications associated with dobutamine stress echocardiography. Experience in 1118 patients. Circulation. 1993;88(1):15-19.

3. Camarozano AC, Siqueira-Filho AG, Weitzel LH, Resende P, Noe RA. The effects of early administration of atropine during dobutamine stress echocardiography: advantages and disadvantages of early dobutamine-atropine protocol. Cardiovasc Ultrasound. 2006;4:17.

4. Lanzarini L, Previtali M, Diotallevi P. Syncope caused by cardiac asystole during dobutamine stress echocardiography. Heart. 1996;75(3):320-321.
5. Hopfenspirger MR, Miller TD, Christian TF, Gibbons RJ. Sinus node deceleration during dobutamine perfusion scintigraphy as a marker of inferior ischemia. Am J Cardiol. 1994;74(8):817-819.

6. Shirasaka W, Ikeshita K, Toriyama S, Yamashita T, Tani Y. [Intraoperative asystole in a patient with concealed sick sinus syndrome: a case report]. Masui. 2014;63(3):338341.

7. Ogawa H, Inoue T, Miwa S, Fujimoto T, Ohnishi Y, Fukuzaki H. Heart rate responses to autonomic drugs in sick sinus syndrome - correlation with syncope and electrophysiologic data. Jpn Circ J. 1991;55(1):15-23.

8. Innes, IR MN. Atropine, scopolamine, and related antimuscarinic drugs. The pharmacological basis of therapeutics. Macmillan New York. 1975;5:514-532.

9. Montano N, Cogliati C, Porta A, Pagani M, Malliani A, Narkiewicz K, Abboud FM, et al. Central vagotonic effects of atropine modulate spectral oscillations of sympathetic nerve activity. Circulation. 1998;98(14):13941399. 